\title{
CARCINOMA OF URINARY BLADDER; EXTENT OF CARCINOMA OF URINARY BLADDER ON FIRST PRESENTATION AND ITS IMPACT ON MANAGEMENT
}

1. MBBS, MS Urology Associate Professor DHQ/GMC Gujranwala

2. MBBS, MS Urology Consultant Urologist Institute of Kidney Disease Hayatabad Medical Complex Peshawa

3. MBBS, MS Urology Assistant Professor DHQ/GMC Gujranwala

4. MBBS, FCPS General Surgery Professor DHQ/GMC Gujranwala

5. MBBS, MS Urology Professor Urology DHQ/GMC Gujranwala

Correspondence Address: Dr. Attiq ur Rahman Khan Assistant Professor DHQ/GMC Gujranwala drattiqkhan@gmail.com

Article received on: 15/06/2017 Accepted for publication: 28/09/2017 Received after proof reading: $03 / 11 / 2017$
Khalid Hussain', Manzoor Ahmad Khan², Attiq ur Rahman Khan³, Prof. Dr. Imran Amin, Prof. Muhammad Khalid Butt ${ }^{5}$

ABSTRACT... Introduction: The most common presentation of carcinoma of urinary bladder is haematuria. Almost $80-90 \%$ of patients with carcinoma of bladder present either with microscopic or gross haematuria and it is mostly intermittent rather than constant. Some patients also complain of irritative voiding symptoms such as frequency, urgency and dysuria. Study Design: Descriptive, case series study. Setting: Department of Urology, DHQ Hospital, Gujranwala, Pakistan. Period: July 2015 to June 2016. Materials \& Methods: Total 30 patients irrespective of age and gender with diagnosis of bladder tumor based on clinical symptoms and supported by laboratory tools like Urine cytology, Ultrasound and IVU were included. The already diagnosed patients of bladder tumor that have been treated with different modalities were excluded. All the tumors were staged according to TNM classification after TURBT and graded on the basis of histopathology. Results: Mean age was $53.17 \pm 16.07 \mathrm{SD}$ years. Males were $25(83.3 \%)$ and females were $5(16.7 \%)$. Male to female ratio is $5: 1$. Twenty eight $(93.3 \%)$ patients out of 30 presented with painless haematuria. 23(73.33\%) patients out of 30 patients were smokers. $09(30 \%)$ patients had Ta, in which G1 was found in 2 patients and G2 in 7 patients. T1 was found in 10 (33.3\%) patients, in which G2 was $5(17.3 \%)$ and G3 was $5(17.3 \%)$ cases. Muscle invasive T2a was diagnosed in 04 patients amongst them G2 and G3 was detected in 2(6.6\%) patients each respectively. T2b was present in 3 patients, which had G1 in $1(3.3 \%)$ and G3 in 2(10\%) patients. T3b G2 was found 01 patient and T3b G3 was detected in $1(3.3 \%)$ patient, T4M1 G3 was present in 2(6.6\%) patients. Conclusion: This study concluded that the bladder tumor is quite common with muscle invasive TCC is more common. Painless hematuria is the commonest presentation and also smoking has a definite association with CA bladder.

Key words: $\quad$ Urinary Bladder Carcinoma, Hematuria, Grading.

Article Citation: Hussain K, Khan MA, Khan A, Amin I, Butt MK. Carcinoma of urinary bladder; Extent of carcinoma of urinary bladder on first presentation and its impact on management. Professional Med J 2017;24(11):1691-1696. DOI: $10.17957 / T P M J / 17.4243$

\section{INTRODUCTION}

Bladder carcinoma is the most common urological malignancy of the genitourinary tract in Pakistan ${ }^{1}$ and the second most common cause of death among genitourinary tumors after prostate cancer. ${ }^{2}$ Incidence of carcinoma of urinary bladder in Pakistan is $0.7 \% .^{3}$ It is the fourth most common cancer in men after prostate, lungs and colorectal accounting for $5.5 \%$ of all cancer cases. ${ }^{4}$ In women it is the eighth most common cancer accounting for $2.3 \%$ of all cancers. ${ }^{5,6}$ The most common presentation of carcinoma of urinary bladder is haematuria. Almost $80-90 \%$ of patients with carcinoma of bladder present either with microscopic or gross haematuria and it is mostly intermittent rather than constant. ${ }^{7,8}$
At the time of first presentation about $80 \%$ of patients with bladder cancers have superficial papillary tumors confined to mucosa (Stage Ta) or the lamina propria (Stage T1) in Europe, ${ }^{9}$ but in a study conducted in Pakistan $53 \%$ of carcinoma of bladder patients have a superficial tumor at first presentation and $47 \%$ transitional cell carcinomas (TCCs) invaded the muscular coat of bladder. ${ }^{6,10}$

The outcome of treatment of bladder cancer depends mainly on the local and systemic extent of the disease. ${ }^{11}$ The most common approach to treat superficial bladder cancer is transurethral resection (TUR) with fulguration of the tumor base. ${ }^{12}$ But even with this treatment up to $80 \%$ of patients will have recurrent tumors often during 
the first year. When treatment protocol includes intravesical chemotherapy after TURBT, it reduces the risk of recurrence. ${ }^{9}$ Patient should be considered for radical cystectomy when there is evidence of the primary tumor and the likelihood of spread into the deeper layers of the bladder. ${ }^{10}$ Cystectomy was highly effective in curing patients with high grade superficial disease including those with superficially invasive disease associated with nodal metastasis. ${ }^{13}$

In our country, patient presentation and diagnosis of the disease is delayed due to ignorance, poverty, lack of health education and poor diagnostic facilities. Therefore the more advanced extent of carcinoma urinary bladder may be seen in our patients as compared to that reported in literature. This project was designed to see the extent of carcinoma urinary bladder at first presentation and its impact on management in terms of hospital stay and cost of treatment.

\section{MATERIALS AND METHODS}

This descriptive, case series study was conducted at the Department of Urology, DHQ Hospital, Gujranwala, Pakistan from July 2015 to June 2016. A sample of 30 patients irrespective of age and gender with diagnosis of bladder tumor based on clinical symptoms and supported by laboratory tools like urine cytology, ultrasound and IVU were included. The already diagnosed patients of bladder tumor that have been treated with different modalities were excluded. Blood complete examination, urine complete examination, urine cytology, blood urea, Serum creatinine, and LFT's were performed in all the cases. All patients were admitted prior to surgery. All the patients underwent chest X-ray PA view, ultrasound abdomen and pelvis, IVU preoperatively while CT abdomen and pelvis liver scan and bone scan were done post operatively for metastatic workup and for staging of the disease.

Twelve hours NPO (nothing per oral) was considered mandatory for all patients undergoing transurethral resection of bladder tumor. All the patients underwent surgery under spinal anesthesia. Patients were placed in lithotomy position after spinal anesthesia. Genitalia (penis \& scrotum in males, labia majora, minora, clitoris and urethra in females), perineum, suprapubic region and upper third of each thigh were thoroughly cleaned with pyodine solution followed by application of sterilized drappings.

After passing cystoscope sheath the specimen of urine was collected for cytology. Bimanual examination was performed before and after resection of tumor in each patient. The procedure started with cystourethroscopy that was carried out with rigid cystoscope of $23 \mathrm{Fr}$ (French) sheaths and $30^{\circ}$ lens telescope. The whole of the urethra and prostatic fossa as well as the entire urethra were carefully and completely inspected. Then cystoscope was removed. Transurethral resection of bladder tumor was performed through a $26 \mathrm{Fr}$ resectoscope using a cutting loop and electrocautery apparatus. The resection was done in a manner that first the tumor then the pedicle and lastly base was resected in addition surrounding deep muscle biopsy was also taken and sent separately. Collected specimens were gently placed in the formalin bottle. The bottle was labeled with patient's name, age, hospital registration number, name of the specimen and the collection date. All patients were catheterized at the end of the procedure, preferably with a large bore (22Fr) 3-way Foley catheter and normal saline irrigation was attached. In all patients, catheter was removed after 4 days.

Aminoglycosidesorthirdgenerationcephalosporin were given immediately before the start of the procedure and postoperatively oral quinolones were continued for 3 days. All the tumors were staged according to TNM classification after going through the above protocol and graded on the basis of histopathology. Patients were then managed according to stage and grade of the disease. All the data was entered and analyzed using SPSS-20. Descriptive statistics were applied to calculate mean and standard deviation for the age of the patients. Frequencies and percentage was calculated for the categorical variables.

\section{RESULTS}

Thirty patients were included in study in the range 
21-80 years with mean age of $53.17+16.07 \mathrm{SD}$ years. Males were $25(83.3 \%)$ and females were $5(16.7 \%)$. Male to female ratio is $5: 1$.

Seven (23.3\%) patients were factory workers, (Textiles, tyre rubber and cable worker) $6(20 \%)$ patients were farmers, $5(16.7 \%)$ patients were house wives, $3(10 \%)$ patients were government servants, $4(13.3 \%)$ patients were shop keeper $1(3.3 \%)$ patient was driver and $4(13.3 \%)$ were field workers. Twenty eight (93.3\%) patients out of 30 presented with painless haematuria and the complication of haematuria as clot retention. Two (6.6\%) patients were presented with the symptoms of frequency and urgency. In the past medical history, 2(6.6\%) patients were having history of diabetes and $3(10 \%)$ patients had hypertension. $3(10 \%)$ patients had history of transvesical prostatectomy. $23(73.33 \%)$ patients out of 30 patients were smokers.

All 30 patients were staged after detailed history, clinical examination and investigations, in which $19(63.3 \%)$ patients were found to have superficial bladder tumor (Ta, T1), 07(23.3\%) patients had muscle invasive bladder tumor (T2a, T2b) and 02 patients had perivesical fat involvement (T3b) while $2(6.6 \%)$ patients had distant metastases (T4M1) as shown in Table I.

Histo-pathological examination showed that 09 (30\%) patients had $\mathrm{Ta}$, in which $\mathrm{G} 1$ was found in

2 patients and G2 in 7 patients. T1 was found in $10(33.3 \%)$ patients, in which G2 was $5(17.3 \%)$ and G3 was $5(17.3 \%)$ cases. Muscle invasive T2a was diagnosed in 04 patients amongst them G2 and G3 was detected in 2(6.6\%) patients each respectively. T2b was present in 3 patients, which had $\mathrm{G} 1$ in $1(3.3 \%)$ and $\mathrm{G} 3$ in $2(10 \%)$ patients. T3b G2 was found 01 patients and T3b G3 was detected in $1(3.3 \%)$ patient, T4M1 G3 was present in 2(6.6\%) patients as shown in Table II.

Check cystourethroscopy was performed at 3 months interval for 2 years then 6 monthly for 1 year and yearly for next 2 years. During follow up, 11(57.8\%) patients had multiple recurrences during first 12 months. Out of 11 patients, recurrence occurred in 6 patients with G3, 4 patients with G2 while only 1 patient with GI had recurrence. Regarding the management of recurrent superficial bladder tumor, following resection of recurrent tumor, Intravesical Immunotherapy in the form of BCG was given in 11 recurrent cases 6 patients with G3 grade and 4 patients with G2 grade and in 01 patient with recurrent $\mathrm{G} 1$.

Intravesical BCG was found successful in controlling the further recurrence over extended period of 12 months in 6 patients (54\%). Those 5 patients who failed to respond to BCG developed progression to muscle invasive disease in 2 cases.

\begin{tabular}{|l|c|c|}
\hline \multicolumn{1}{|c|}{ Clinical Staging } & Frequency & Percent (\%) \\
\hline Superficial bladder cancer (Ta, T1) & 19 & 63.3 \\
\hline Invasive bladder tumor ( T2a, T2b) & 07 & 23.3 \\
\hline Locally advance disease(T3b) & 02 & 6.6 \\
\hline Metastatic bladder tumor (T4M1) & 02 & 6.6 \\
\hline Total & 30 & 100.0 \\
\hline
\end{tabular}

Table-l. Clinical staging of the urinary bladder carcinoma $(n=30)$

\begin{tabular}{|c|c|c|c|c|c|}
\hline Clinical Stage & No of Patients & \multicolumn{2}{|c|}{ Histological Grades } & Total \\
\hline Ta & 09 & 02 & 07 & 00 & 09 \\
\hline T1 & 10 & 00 & 05 & 05 & 10 \\
\hline T2 a & 04 & 00 & 02 & 02 & 04 \\
\hline T2 b & 03 & 01 & 00 & 02 & 03 \\
\hline T3b & 02 & 00 & 01 & 01 & 01 \\
\hline T4 M1 & 02 & 00 & 00 & 02 & 02 \\
\hline Total & 30 & 08 & 15 & 07 & 30 \\
\hline
\end{tabular}




\section{DISCUSSION}

Bladder tumor is a common illness presenting to urological discipline across the globe. It is associated with significant mortality as well as morbidity. Although there has been tremendous research in early detection of TCC bladder and break through has been established in controlling the clinical risk factors in terms of preventing recurrences resulting in improvement in quality of life, lesser hospital stay and better cost effectiveness. Bladder tumor usually affects the patients in $5^{\text {th }}$ and $6^{\text {th }}$ decade of life. In our study the mean age was 53.17years+16.07SD. The mean age of presentations of carcinoma of bladder was 67 years, in a study conducted by Chinegwundoh et al in 1996. ${ }^{14}$

In our study, there were 25 (out of 30) males and 5 females patients. In this study male to female ratio was about to $5: 1$ which shows that this tumor is more common in males than in females. While according to other statistics on this subject, males are affected approximately 2.5 to 4 times as often as females, with male to female ratio of $2: 1$ and $3: 1 .^{6}$

This ratio was somewhat closer to that of American $^{7,8}$ and Australian studies. ${ }^{15}$ The comparatively high incidence in males (in our study) may be due to the more exposure to different carcinogens in relation to smoking and occupational exposure because in our set-up females are restricted to home and it is the male who earns the living. Smoking in female is also very rare in our part of world. Women also come to the doctor late in their stage of the disease mainly due to social taboos while men seek treatment earlier. $^{16}$

The hallmark symptom of bladder tumor is painless haematuria. This fact was also noticed in present study. Twenty eight (93.3\%) patients out of 30 presented with gross haematuria, with intermittent passage of clots per urethra at the same time. According to other studies done on clinical presentation of bladder cancer, painless haematuria is the main presenting symptom in $80-90 \%$ of patients. ${ }^{7}$ Frequency and urgency were observed in $2(6.6 \%)$ patients, dysuria was found in $2(6.6 \%)$. In literature $15-20 \%$ patients presented with LUTS. ${ }^{17}$

The results of our study regarding clinical presentation are quite comparable with those of other studies already carried on bladder tumors. Ninety three percent of patients in this study presented with haematuria. This is comparable to world literature were haematuria is presenting symptoms in $80-90 \%$ cases. The reason could be that blood in urine is considered very dangerous in our set-up in comparison to other urinary symptoms like frequency, urgency and dysuria that is why $93.3 \%$ of patients in this study came to the hospital/clinic with haematuria as the $1^{\text {st }}$ presenting symptom. Although some of them had frequency, urgency and dysuria before haematuria.

It is very astonishing that the mean delay in seeking medical treatment after first episode of haematuria is 36 weeks. This delayed presentation is attributed to poor health care and referral system in Pakistan as most of these patients are usually treated as UTIs with multiple antibiotics by GPs and Quacks.

Bladder tumor is recognized as an occupational hazard in international arena. Although direct exposure to carcinogens can be observed in our $7(23.3 \%)$ patients who were factory workers, (Textiles, tyre rubber and cable worker) however indirect exposure to carcinogen was present in $6(20 \%)$ patients who were farmers and were exposed indirectly to insecticides and 1 patient who was heavy traffic vehicle driver was exposed to gasoline products.

These all findings are quite comparable to other studies according to which about $20 \%$ of bladder cancer is caused by direct occupational exposure. ${ }^{1}$ So we reached at a conclusion that factory workers (Textiles, tyre rubber and cable worker) are at high risk for CA urinary bladder in our country. A lot of other carcinogens or highrisk occupations are still to be detected apart from those present in the literature..$^{9,16}$

Smoking is very important factor in causation of 
CA bladder. Cigarette smokers have up to 4 fold higher risk of CA bladder than non-smokers. ${ }^{16}$ The more the number of cigarettes smoked and the more the duration of smoking, the higher the risk. ${ }^{9}$ The specific chemical carcinogen in tobacco is yet to be identified, but some workers have suggested that 2-naphthylamine may be responsible for bladder cancer. Stopping smoking improves the prognosis by reducing the incidence and recurrence. At the time of diagnosis smokers are 3 years younger than nonsmokers. As many as $50 \%$ cases of bladder cancer are attributed to smoking in men and $33 \%$ in women. ${ }^{1}$

In our study out of 30 patients 23 (75.9\%) patients were smokers. The incidence in our study of male smoker is $75.9 \%$, which is comparable to that of $65 \%$ of bladder cancer in men is attributed to cigarette smoking. ${ }^{16}$ Haemoglobin less than $10 \mathrm{gm} / \mathrm{dl}$ was found in $11(36.6 \%)$ cases. Other hematological and biochemical investigations including RFTs and LFTs were normal. According to other studies, anemia is occasionally present unless gross episodes of haematuria. ${ }^{7}$ This fact was also observed in our study as 28 of my patients came to the hospital with gross haematuria.

In our study 19 (63.3\%) had superficial bladder cancer, while the muscle invasive and locally advance TCC bladder on $1^{\text {st }}$ presentation were $11(30 \%)$ patients. In literature incidence of superficial bladder cancer is $80 \%$. While invasive bladder tumor on first presentation is $15-25 \% .^{9}$ Although this observation in contradictory to international literature but the more prevalence of invasive bladder tumor can be sighted in national and regional literature. ${ }^{18}$ The possible reason for this discrepancy could be the delayed presentation of our patients to physicians due to lack of education, ignorance and financial constraints.

Any surgical treatment has potential hazards which results in morbidity and even mortality and this is true for TURBT also which is not spared of complications. Early complications are massive hemorrhage and clot retention. ${ }^{9}$ In our study, 4 patients developed secondary hemorrhage, which was treated conservatively with catheterization, bladder wash, blood transfusion and antibiotics. In 2(6.6\%) cases, clot retention was observed which was treated by bladder wash. None of the patient got bladder perforation and mortality. These complications are never more than those present in literature and they can be reduced with expertise of the urologist. ${ }^{19}$

Our study is implicated on the Urologists who are involved in initial management of Bladder tumors. More over as TCC is occupational hazard it is also implicated on public health authorities and legislative bodies for implementation of rules regarding compensation for the workers in different industries. We have observed that our patients present very late as compared to western hemisphere. In our country, patient presentation and diagnosis of the disease is delayed more importantly due to ignorance of the early symptoms by patient, poverty, lack of health education and poor diagnostic facilities. Therefore more advanced extent of carcinoma urinary bladder may be seen in our patients as compared to that reported in literature. The significant delay in diagnosis results in more aggressive and high stage disease and treatment is palliative with poor prognosis and increased financial burden due to prolonged hospital stay. This project had designed to see the extent of carcinoma urinary bladder at first presentation and its impact on management in terms of hospital stay and cost of treatment.

\section{CONCLUSION}

This study concluded that the bladder tumor is quite common with muscle invasive TCC is more common. Painless hematuria is the commonest presentation and also smoking has a definite association with CA bladder. So, we recommend that proper approach should be adopted in all patients presenting with urinary bladder cancer in order to reduce the morbidity and mortality of these particular patients.

Copyright (C) 28 Sep, 2017.

\section{REFERENCES}

1. Rafique M, Javaid M, Abrar A. Clinico-pathological feature of bladder carcinoma: experience from a tertiary care hospital of Pakistan. Intl Urol Nephrol. 
2006;38(2):247-50.

2. Marc-Oliver G, Steinhoff C, Simon, X. Effect of routine repeat transurethral resection for superficial bladder cancer: A long term observational study. J Urol. 2003;170:433-7.

3. Memon AR. Pattern of malignant tumours seen at Dow Medical College and Civil Hospital, Karachi. Pak J Med Sci Pak Med Sci. 2000;16:129-34.

4. Friedrich MG, Hellstern A, Hautmann SH. Clinical use of urinary markers for the detection and prognosis of bladder carcinoma: A comparison of immunocytology with monoclonal antibodies against lexis $X$ and $486 \mathrm{p} 3 / \mathrm{i2}$ with the BTA stat and NMP22 tests. J Urol. 2002;168(2):470-6.

5. Boring CC, Squires TS, Tang T. Cancer statistics 1995. Caner J Clin. 1995;45:2.

6. Siyal AR, Shaikh SM, Jalbani MH. A clinicopathological study 108 cases of urinary bladder cancer at Chandka Medical College \& Hospital, Larkana. J Surg Pak (International). 1999;4:7-9.

7. Khadra MH, Pickard RS, Charitom M. A prospective analysis: 1930 patients with heamaturia to evaluate current diagnostic practice. J Urol. 2000;163:524.

8. Messing EM, Young TB, Hunt YB. Hematuria home screening: Repeat testing results. Urol. 1995;154:5761.

9. Sylvester RJ, Vandermeijden APM, Lamm DL. Intravesical bacillus Calmette-Guerin reduces the risk of progression in patients with superficial bladder cancer: a meta-analysis of the published results of randomized clinical trials. J Urol. 2002;168:1964.

10. Stein JP, Ginsberg DA, Groosfeld GD. Effect of P21 waf $1 /$ Cipl: Expression on tumour, progression in bladder cancer. J Natl Cancer Inst. 1998;90:1072-9.
11. Mameghan H, Fisher R. Invasive bladder cancer, prognostic factors and result of radiotherapy with and without cystectomy. Br J Urol. 1989;63:258.

12. Synder C, Harlan L, Knopf K. Patterns of care for the treatment of bladder cancer. Am J Urol. 2003;169:1697701.

13. Weizer AZ, Joshi D, Diagnault S. Performance status is a predictor of overall survival of elderly patient with muscle invasive bladder cancer. $\mathrm{J}$ Urol. 2007; 177:1287-93.

14. Chinegwundoh F, Kaisary AV. Polymorphism and smoking in bladder carcinogenesis. $\mathrm{Br} J$ Urol. 1996;77:672.

15. Tekes A, Kamal IR, Chan TY. MR imaging features of non-transitional cell carcinoma of the urinary bladder with pathologic correlation. AJR Am Roentgenol. 2003; 180:779-84.

16. Samanic C, Kogevinasm M, Dosemeci M. Smoking and bladder cancer in Spain: Effects of tobacco type, timing, environmental tobacco smoke, and gender. Cancer Epidemiol Biomarkers Prevention. 2005;15:1348-54.

17. Anjos DA, Elba CSC, Etchebehere CDR. ${ }^{18}$ F-FDG PET/CT delayed images after diuretic for restaging invasive bladder cancer. J Nuclear Med. 2007;48(5):764-70.

18. Ather $\mathbf{M H}$, Fatima $\mathrm{S}$, Sinanoglu $\mathrm{O}$. Extent of lymphadenectomy in radical cystectomy for bladder cancer. World J Surg Oncol. 2005;1477-7819.

19. Kikuchi E, Horiguchi $Y$. Assessment of long-term quality of life using the FACT-BL questionnaire in patients with an Ileal Conduit, Continent Reservoir, or Orthotopic Neobladder. Japanese J Clin Oncol. 2006;36(11):712-6.

\section{AUTHORSHIP AND CONTRIBUTION DECLARATION}

\begin{tabular}{|c|c|c|c|}
\hline Sr. \# & Author-s Full Name & Contribution to the paper & Author $=$ s Signature \\
\hline 1 & Khalid Hussain & Main Resercher & \\
\hline 2 & Manzoor Ahmad Khan & Data collection & \\
\hline 3 & Attiq ur Rahman Khan & $\begin{array}{l}\text { Making performa and } \\
\text { statistic analysis }\end{array}$ & 2 \\
\hline 4 & Prof. Dr. Imran Amin & Proof reading & \\
\hline 5 & Prof. M. Khalid Butt & Supervisor & \\
\hline
\end{tabular}

